\title{
Hepatocellular Carcinoma Showing Pathological Complete Response to Lenvatinib Monotherapy
}

\author{
Hiroshi Shintani Shoji Oura Shinichiro Makimoto \\ Department of Surgery, Kishiwada Tokushukai Hospital, Kishiwada City, Japan
}

\section{Keywords}

Hepatocellular carcinoma · Lenvatinib · Pathological complete response

\begin{abstract}
A 61-year-old man was referred to our hospital due to the liver dysfunction without hepatitis $\mathrm{B}$ or $\mathrm{C}$ infection. In addition to the elevated levels of $\alpha$-fetoprotein (AFP) and protein induced by vitamin $\mathrm{K}$ absence or antagonist-II, a large tumor, $10.8 \mathrm{~cm}$ in size, and multiple small tumors, up to $1.6 \mathrm{~cm}$ in size, in the liver on computed tomography (CT) led to the diagnosis of unresectable advanced hepatocellular carcinoma (HCC). Levatinib monotherapy resulted in complete disappearance of the small liver tumors and marked shrinkage of the largest tumor with complete disappearance of intratumoral enhancement on $\mathrm{CT}$ and normalization of serum AFP levels. After 2 months' cessation of lenvatinib monotherapy due to side effects, the patient underwent residual tumor resection. The pathological findings showed no viable tumor cells, i.e. pathological complete response. The patient was discharged from the hospital on the twelfth day after the operation without any complication. Lenvatinib monotherapy appears to be more effective for HCC than other conventional treatments. In addition, oncologists should take into consideration the possibility of pathological complete response with newly developed anticancer agents including lenvatinib to develop therapeutic strategies to avoid unnecessary overtreatment.
\end{abstract}

\section{Introduction}

Hepatocellular carcinoma (HCC) is the sixth most frequently diagnosed cancer worldwide and is the second leading cause of tumor-related death. Due to both its biological aggressiveness and difficulties in image diagnosis, HCCs are often diagnosed late, leading to a median survival of 6-20 months [1].

\section{Karger"}


Chemotherapy has long been one of the main options in the treatment of advanced HCCs despite its nominal or limited efficacy against large HCCs. The chemorefractory nature of HCC is at least partly due to the overexpression of p-glycoprotein, glutathione S-transferase, and heat shock proteins, and to the mutations in p53. To overcome this chemoresistance in the treatment of advanced HCCs, chemotherapy is often concurrently administered with transarterial embolization.

In recent years, various molecular-targeted drugs and immune checkpoint inhibitors have dramatically changed the therapeutic strategy of advanced HCCs. We present a case of advanced HCC showing clinical and pathological complete response to lenvatinib monotherapy.

\section{Case Report}

A 61-year-old man was referred to our hospital due to the liver dysfunction without hepatitis B or C infection. Contrast enhanced computed tomography (CT) showed a liver tumor $108 \times 102 \mathrm{~mm}$ in size in S7 and multiple liver tumors up to $1.6 \mathrm{~cm}$ in diameter in the liver without apparent vessel invasion (Fig. 1a). Tumor marker levels of $\alpha$-fetoprotein (AFP) and protein induced by vitamin $\mathrm{K}$ absence or antagonist-II (PIVKA-II) were significantly increased to 81.6 (normal range: $0-10$ ) $\mathrm{ng} / \mathrm{mL}$ and 112,775 (normal range: $0-40$ ) $\mathrm{mAU} / \mathrm{mL}$, respectively. Under the diagnosis of unresectable advanced HCC, the patient underwent lenvatinib monotherapy ( $8 \mathrm{mg}$ per day). His serum AFP and PIVKA-II levels decreased to 18.3 $\mathrm{ng} / \mathrm{mL}$ and $20,729 \mathrm{mAU} / \mathrm{mL}$ only in 1 week, and further to $6.2 \mathrm{ng} / \mathrm{mL}$ and 4,207 $\mathrm{mAU} / \mathrm{mL}$ in 4 weeks, respectively. The patient developed manageable hand-foot syndrome, hypertension, and hypothyroidism. His serum AFP level eventually normalized to $5.6 \mathrm{ng} / \mathrm{mL}$ in 4 months and continued to show the normal values thereafter, whereas serum PIVKA-II levels fluctuated around $100 \mathrm{mAU} / \mathrm{mL}$. Five months after the initiation of lenvatinib monotherapy, contrast-enhanced CT showed the largest tumor to be $71 \times 69 \mathrm{~mm}$ in size with no intratumoral enhancement and complete disappearance of the bilateral small multiple liver lesions

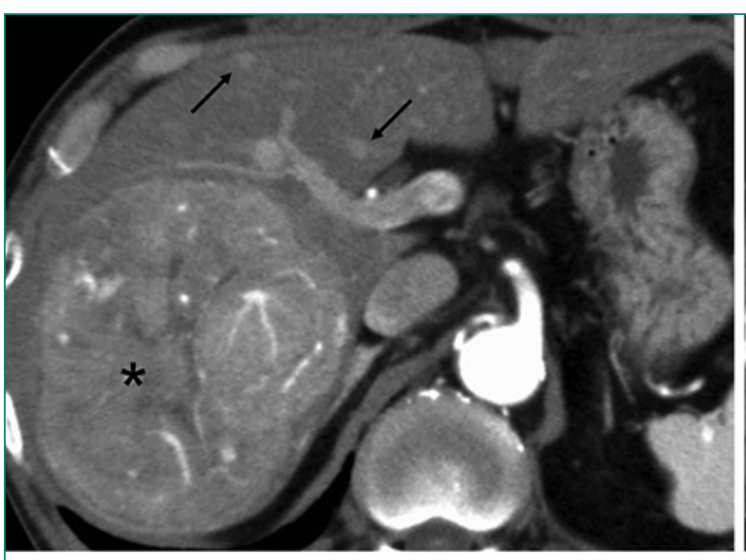

a

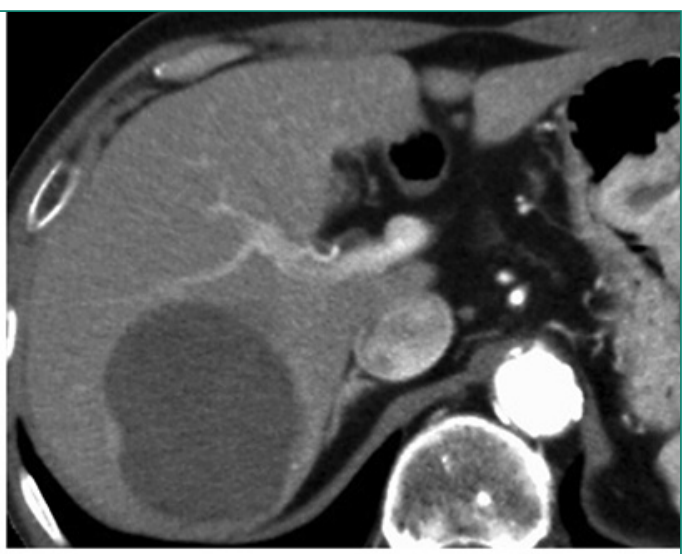

b

Fig. 1. Enhanced computed tomography of the liver. a Before lenvatinib treatment. In addition to the largest tumor with intratumoral enhancement (asterisk), small enhanced nodules (arrows) were observed. b After 12 months of lenvatinib treatment. The largest tumor regressed markedly without intratumoral enhancement. In addition, small nodules disappeared completely. 


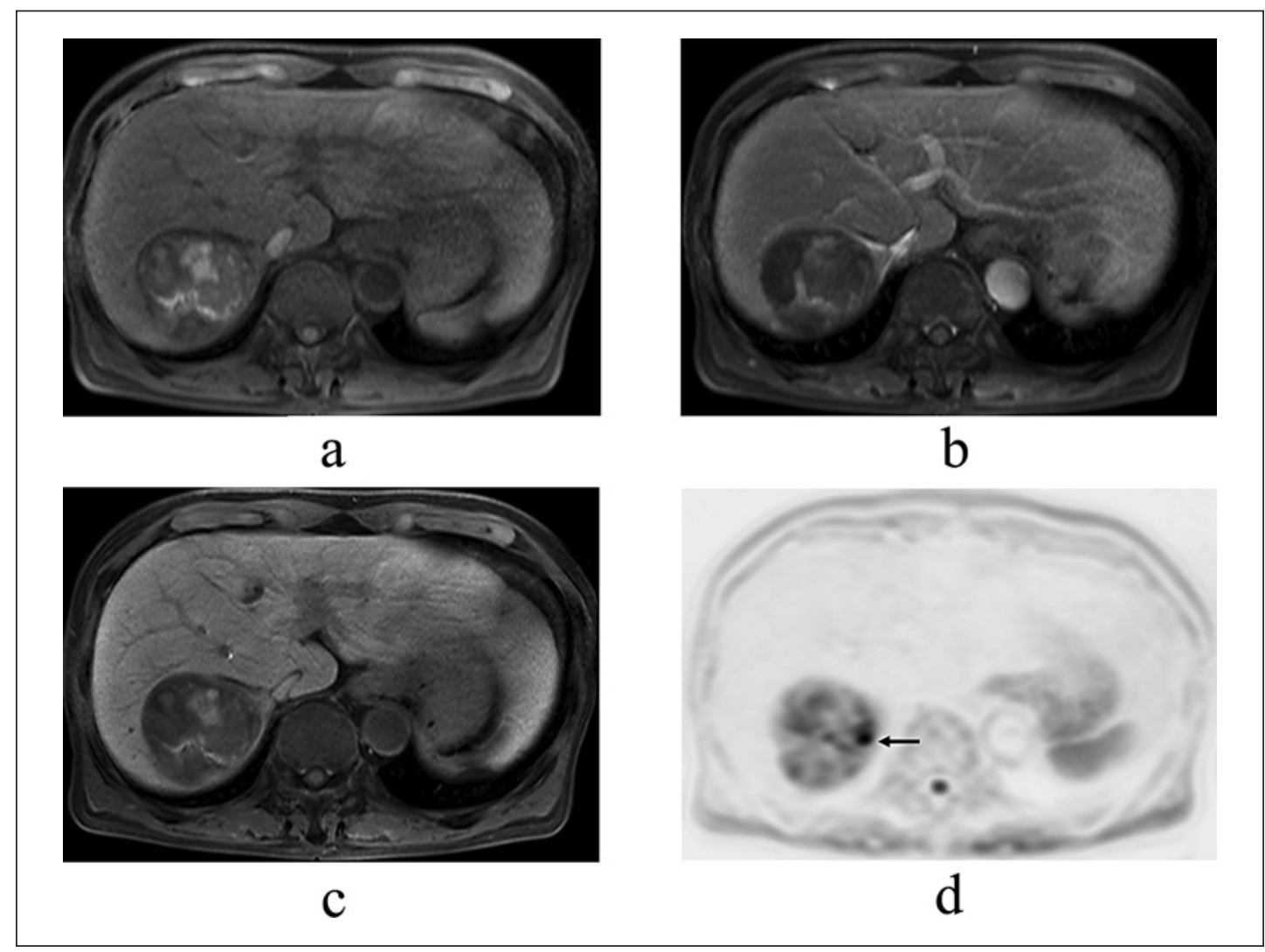

Fig. 2. Magnetic resonance imaging just before the operation. a T1 weighted image without contrast agent. A well-demarcated oval mass with internal patchy and striated hyperintense areas was observed. b Early phase T1 weighted image after contrast agent administration. No tumor enhancement was observed. c Hepatobiliary phase T1 weighted image after contrast agent administration. Normal liver parenchyma was enhanced, but no enhancement was observed in the tumor. $\mathbf{d}$ Diffusion weighted image. A focal hyperintense signal (arrow) was observed in the tumor.

(Fig. 1b). Favorable response lasted for 12 months with the lenvatinib monotherapy. He, however, discontinued lenvatinib monotherapy due to the side effects, i.e. diarrhea and appetite loss, leading to his hospitalization. After 2 months of off-treatment, CT showed neither tumor regrowth nor new liver lesions. Ultrasonography showed that the main tumor was $73 \times 56 \mathrm{~mm}$ in size with halo, internal mosaic pattern, and no intratumoral blood flow. Magnetic resonance imaging also showed a well-demarcated mass with internal patchy and striated hyperintense areas on T1-weighted images (T1WI; Fig. 2a), no tumor enhancement both on early and hepatobiliary phase T1WI with subtraction (Fig. 2b and c), and a focal hyperintense signal on diffusion-weighted images (DWI; Fig. 2d). The patient was referred to our Department for residual tumor resection. For the pathological assessment and complete local control, the patient underwent S7 subsegmental hepatectomy. Pathological study revealed an encapsulated tumor with massive hyalinization, necrosis, cholesterin crystals, lymphocyte and granulocyte infiltration, and no viable tumor cells (Fig. 3). The patient was discharged 12 days after the operation without any complication. 


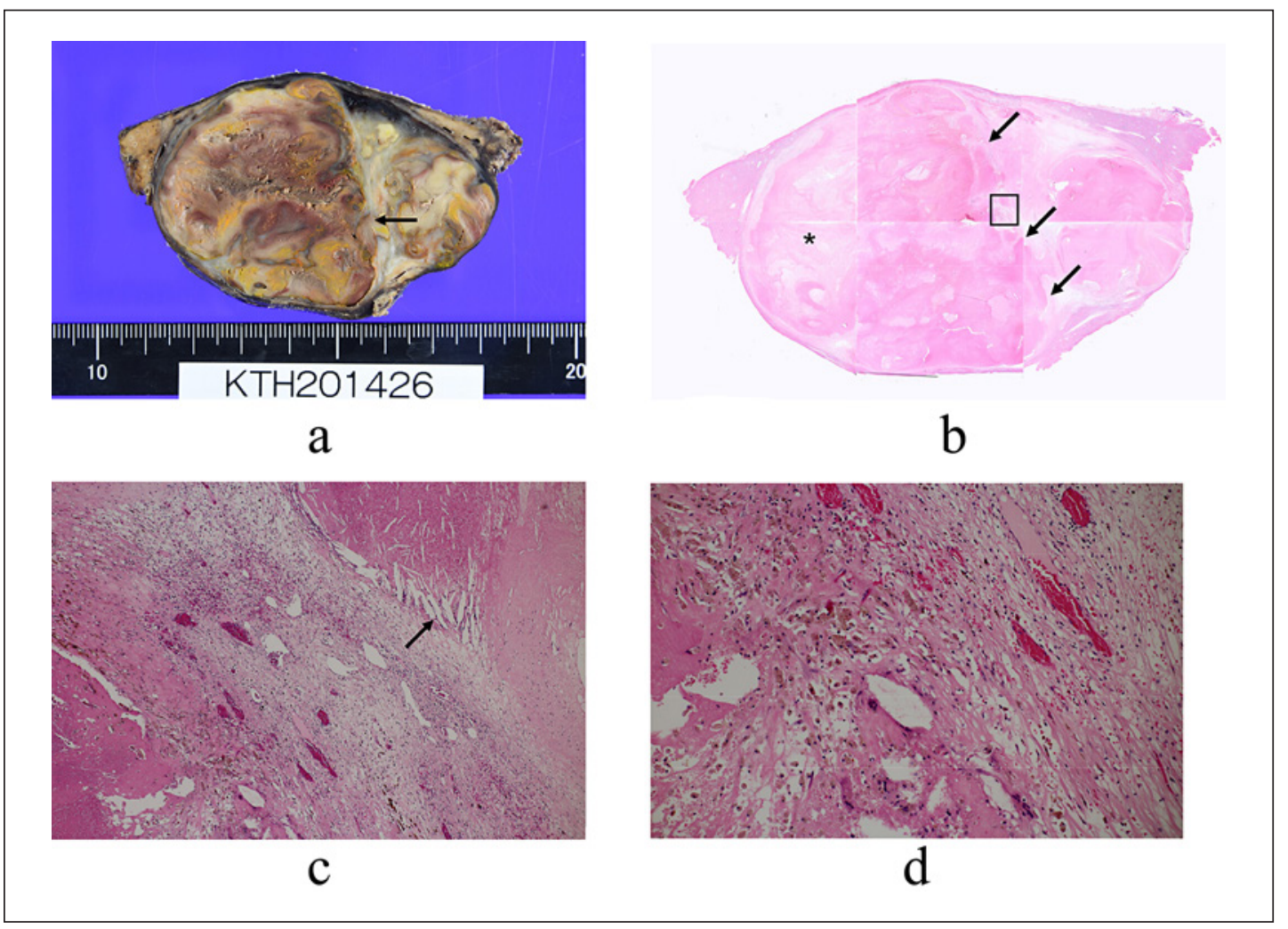

Fig. 3. Pathological findings. a Macroscopic findings of the tumor. A well-demarcated mass was observed. Large brownish and small whitish parts were apparently partitioned (arrows). b Low magnification view. Judged by the correlation among the cut surface of the tumor, the low magnified pathological view, and the magnetic resonance imaging (MRI), the area with low cellular density in the left (asterisk) and the fibrous layer (arrows) corresponded to the focal patchy and striated hyperintense areas on MRI (Fig. 2a), respectively. c Magnified view of the square in Fig. 1b. No viable tumor cells and cholesterin crystals (arrow) were seen. d Magnified view of Fig. 1c. Loosely distributed collagens, lympohocytes, granulocytes, and erythrocytes were observed.

\section{Discussion}

Surgical resection can give the best survival benefit to the patients with resectable HCCs [2-6]. However, due both to the aggressiveness of HCCs and poor hepatic function reserve in many patients with underlining chronic liver disease, limited number of patients with HCC are amenable to surgical intervention. On the other hand, chemotherapy has long been a feasible therapeutic option especially for patients with advanced HCC. Standard chemotherapeutic regimen, however, has not yet been established due to its limited efficacy against HCCs. Therefore, the 5-year survival rate of the patients with HCCs is extremely dismal (2\%) [7].

In 2008, the phase III SHARP trial showed a survival benefit of sorafenib, an oral multikinase inhibitor of the vascular endothelial growth factor receptor, the platelet-derived growth factor receptor, and Raf, compared with that of best supportive care [8]. Thereafter, sorafenib has been the mainstay in the treatment of advanced HCCs. The REFLECT trial using lenvatinib further showed much higher response rate, i.e. 40.6\%, than that of sorafenib [9]. Lenvatinib monotherapy, therefore, has become one of the feasible first-line therapies for advanced HCCs. 
Various advanced solid malignancies are often treated with neoadjuvant therapy using some antitumor agents \pm radiotherapy followed by local therapy, mainly surgery, when marked tumor shrinkage with the neoadjuvant therapy enables surgeons to resect the regressed tumor(s). However, due both to the marked surgical invasiveness of hepatectomy and high rate of recurrence in the liver, pathological response of the regressed HCCs are seldom evaluated even when neoadjuvant therapy brings about marked tumor response. This case, therefore, is extremely rare in view of the pathologically confirmed complete response of advanced HCC.

It is often experienced in the treatment of advanced breast cancer that prompt tumor response to neoadjuvant therapy leads to the pathological complete response. In this case, lenvatinib monotherapy brought about marked decrease in tumor marker levels only in 1 week. We should investigate the correlation between the final antitumor effect and the response speed in patients with HCCs. Persistent mass with no tumor enhancement both on CT and MRI and a hyperintense signal on DWI, mildly elevated PIVKA-II levels, and the need to discontinue lenvatinib monotherapy with adverse effects made it hard for us to manage the residual focus.

In this case, a focal hyperintense signal on DWI urged us to move on to the surgical intervention. However, it is difficult for physicians to determine the therapeutic strategy of the advanced HCC patients with favorable response to some anticancer therapy among three options: (1) to continue some kind of systemic therapy; (2) to proceed to hepatectomy or some kind of ablation; or (3) to be on careful observation without any therapy. If we had judged the residual tumor not by Response Evaluation Criteria in Solid Tumor (RECIST) v.1.1 but by modified RECIST [10], we could have carefully followed the presumed nonviable mass without any antitumor therapy. On the other hand, Liu et al. [11] reported a similar case of multiple HCCs with temporary complete response, except for one small lesion, to lenvatinib monotherapy, finally leading to unresectable regrowth of the residual small HCC. Residual and resectable HCC(s) judged as viable with modified RECIST should be treated with, if possible, some kind of local therapy to avoid undertreatment.

Unfortunately, MRI evaluation of the liver tumors was not done before lenvatinib monotherapy. We, therefore, could not evaluate how MRI findings of the largest HCC changed with lenvatinib monotherapy. MRI, however, depicted the details of the intratumoral components much better than plain and enhanced CT. On the other hand, DWI misled us to surgical intervention in this case. Oncologists should note high signal intensity on DWI can be only based on relative difference of diffusing capacity among various components in and around the tumor, leading to possible misunderstanding of the tumor viability.

In conclusion, we experienced a case of unresectable HCC showing pathological complete response to lenvatinib monotherapy. We should further investigate the proper indication of lenvatinib monotherapy for patients with advanced HCC in comparison with bevacizumab plus athezolizumab combination therapy.

\section{Statement of Ethics}

We have reported this case in compliance with the Declaration of Helsinki. Informed written consent was obtained from the patient for the publication of the clinical data.

\section{Conflict of Interest Statement}

The authors have no conflicts of interest to declare.

\section{Karger'}




\section{Case Reports in Oncology}

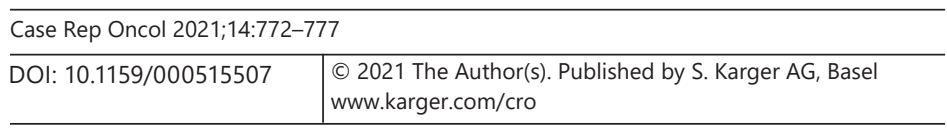

Shintani et al.: HCC Showing Complete Response to Lenvatinib Monotherapy

\section{Funding Sources}

No funding was received for this research.

\section{Author Contributions}

H. Shintani contributed the design of the report. S. Oura drafted the manuscript. S. Makimoto revised the manuscript. All authors have read and approved the final version of the manuscript.

\section{References}

1 The cancer of the liver Italian program (CLIP) investigators. A new prognostic system for hepatocellular carcinoma: a retrospective study of 435 patients: the Cancer of the Liver Italian Program (CLIP) investigators. Hepatology. 1998;28(3):751-5.

2 Chawla A, Ferrone C. Hepatocellular carcinoma surgical therapy: perspectives on the current limits to resection. Chin Clin Oncol. 2018;7(5):48-55.

3 Llovet JM, Brú C, Bruix J. Prognosis of hepatocellular carcinoma: the BCLC staging classification. Semin Liver Dis. 1999;19(3):329-38.

4 Llovet JM, Fuster J, Bruix J. Intention-to-treat analysis of surgical treatment for early hepatocellular carcinoma: resection versus transplantation. Hepatology. 1999;30(6):1434-40.

5 Chok KS, Ng KK, Poon RT, Lo CM, Fan ST. Impact of postoperative complications on long-term outcome of curative resection for hepatocellular carcinoma. Br J Surg. 2009;96(1):81-7.

6 Kianmanesh R, Regimbeau JM, Belghiti J. Selective approach to major hepatic resection for hepatocellular carcinoma in chronic liver disease. Surg Oncol Clin N Am. 2003;12(1):51-63.

7 Siegel RL, Miller KD, Jemal A. Cancer statistics, 2016. CA Cancer J Clin. 2019;66(1):7-30.

8 Llovet JM, Ricci S, Mazzaferro V, Hilgard P, Gane E, Blanc JF, SHARP Investigators Study Group, et al. Sorafenib in advanced hepatocellular carcinoma. N Engl J Med. 2008;359(4):378-90.

9 Kudo M, Finn RS, Qin S, Han KH, Ikeda K, Piscaglia F, et al. Lenvatinib versus sorafenib in first-line treatment of patients with unresectable hepatocellular carcinoma: a randomised phase 3 non-inferiority trial. Lancet. 2018;391(10126):1163-73.

10 Lencioni R, Llovet JM. Modified RECIST (mRECIST) assessment for hepatocellular carcinoma. Semin Liver Dis. 2010;30(1):52-60.

11 Liu Z, Fu Z, Li G, Lin D. Downstaging of recurrent advanced hepatocellular carcinoma after lenvatinib treatment: Opportunities or pitfalls? A case report. Onco Targets Ther. 2020;13:10267-73. 